\title{
Médiévales
}

Langues, Textes, Histoire

47 | automne 2004

Îles du Moyen Âge

\section{Jean-Claude Schmitt, Le corps des images. Essais sur la culture visuelle au Moyen Âge}

Paris, Gallimard, Collection « Le temps des images », 2002, 410 p.

\section{Christopher Lucken}

\section{(2) OpenEdition}

\section{Journals}

Édition électronique

URL : https://journals.openedition.org/medievales/1085

DOI : 10.4000/medievales. 1085

ISSN : 1777-5892

\section{Éditeur}

Presses universitaires de Vincennes

Édition imprimée

Date de publication : 1 décembre 2004

Pagination : 178-184

ISBN : 2-84292-157-7

ISSN : 0751-2708

\section{Référence électronique}

Christopher Lucken, « Jean-Claude Schmitt, Le corps des images. Essais sur la culture visuelle au Moyen Âge », Médiévales [En ligne], 47 | automne 2004, mis en ligne le 13 mars 2006, consulté le 22 avril 2022. URL : http://journals.openedition.org/medievales/1085; DOI : https://doi.org/10.4000/medievales. 1085

Ce document a été généré automatiquement le 22 avril 2022

Tous droits réservés 


\title{
Jean-Claude Schmitt, Le corps des images. Essais sur la culture visuelle au Moyen Âge
}

Paris, Gallimard, Collection « Le temps des images », 2002, 410 p.

\author{
Christopher Lucken
}

1 Cet ouvrage réunit onze études publiées dans différents lieux entre 1987 et 2000, précédées d'une introduction. Celles-ci ne sont pas classées par ordre chronologique, mais regroupées en fonction de leur sujet en trois parties. La première, «Une longue histoire », porte sur la fonction pédagogique accordée à l'image par le pape Grégoire le Grand, principalement, sur son histoire au Moyen Âge et sur les conséquences qu'une telle conception a pu avoir sur le rôle que les historiens modernes ont réservé à cet objet. La deuxième partie est consacrée au «Culte des images» qui s'affirme principalement à partir de l'an mil. La troisième partie, intitulée "Rêves, visions, fantasmes ", est consacrée à un sujet qu'apprécie particulièrement J.-Cl. Schmitt et sur lequel il a déjà publié de nombreux travaux. Elle aborde ces images qui, sans être à proprement parler des images achéiropoiètes, n'ont pas été réalisées par la main ; mais elle s'intéresse aussi à la manière dont ces images intérieures ont été représentées au moyen d'images réelles.

2 Cette succession d'études se présente comme un ensemble ordonné et cohérent qui en fait un véritable livre. Le parcours qu'il retrace est celui du destin médiéval des images : il commence par étudier les arguments avancés par Grégoire le Grand pour justifier leur emploi dans les églises, pour aboutir à la manière dont les images vont déborder ce cadre théorique et imposer leur présence singulière: que ce soit dans l'espace institutionnel de l'Église, où la vénération que l'on porte désormais aux images religieuses vient concurrencer la primauté octroyée habituellement à la lecture des saintes Écritures, ou dans l'espace mental des hommes, où l'imagination affirme son pouvoir à côté d'une raison dominée par le langage et dont la présence apparaît généralement comme le garant du (bon) fonctionnement de la pensée. Ce parcours est aussi celui effectué par leur auteur. Emblématique, à cet égard, est la modification du 
titre de l'article le plus ancien : alors qu'il était intitulé en 1987 «L'Occident, Nicée II et

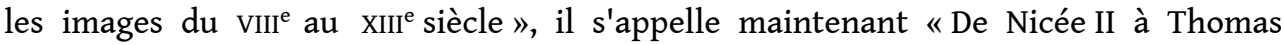
d'Aquin : l'émancipation de l'image religieuse en Occident ». On est passé d'une formule statique se contentant de situer les limites chronologiques de son sujet, à un titre dynamique qui met en valeur une transformation, présentée ici comme un affranchissement. Cette "émancipation de l'image religieuse en Occident» se manifeste principalement sous la forme d'une incarnation : de Grégoire le Grand au XIII siècle, l'image se libère de la lettre à laquelle elle est constamment ramenée pour prendre corps d'une façon qui lui est propre et fonctionner de manière plus ou moins autonome. Mais cette « émancipation » est aussi celle qu'acquièrent les images auprès des historiens modernes: alors qu'elles étaient généralement étudiées à l'aide des textes qui pouvaient en expliquer la signification, elles deviennent désormais un objet d'histoire à part entière. C'est là aussi, peut-être, une manière de contrer certains effets du structuralisme ou du « linguistic turn » dans le champ des études historiques.

Plutôt que de reprendre, ici, l'une après l'autre les différentes études qui ponctuent cette histoire, et ne pas pouvoir rendre compte de toute leur richesse, je préfère en retracer le mouvement d'ensemble, avant d'en interroger rapidement les aboutissants.

La thèse de Grégoire le Grand est exposée principalement dans les deux lettres qu'il adressa en 599 et 600 à l'évêque Serenus de Marseille. Alors que ce dernier avait fait détruire les images peintes aux murs des lieux consacrés au culte, de crainte que les fidèles ne soient tentés de les considérer comme des idoles et se mettent à leur consacrer des rites, Grégoire le Grand affirme que «les images doivent être placées dans les églises, afin que ceux qui ne savent pas les lettres lisent toutefois en regardant sur les parois ce qu'ils ne peuvent lire dans les livres ». Cet argument repose sur une comparaison implicite entre les lettres écrites dans un livre et les images peintes aux parois d'une église. C'est pourquoi, notamment pour celui qui est étranger à la foi, la peinture tient le rôle de la lecture: Pictura est quaedam litteratura illiterato, comme le souligne deux siècles plus tard Walafrid Strabon dans une formule synthétique dont on connaît la fortune. La vision d'une peinture paraît dès lors pouvoir se substituer à la lecture de la Bible. Grâce à elle, les illettrés pourront prendre connaissance d'un enseignement qui, autrement, leur resterait inaccessible.

5 L'image doit permettre en particulier de connaitre l'histoire passée qui s'y trouve représentée, notamment l'histoire biblique et la vie des saints. Comme le souligne J.$\mathrm{Cl}$. Schmitt, "la fonction de l'image est définie par référence à un genre de la littérature écrite : l'histoire, ou plus précisément l'historia, l'histoire sainte, celle de la vie et de la mort du Christ et des saints martyrs. Cette histoire doit être peinte (depingi historias), pour devenir l'histoire figurée en peinture (picturae historia) offerte à la vue (visio historiae, ex visione rei gestae) [...]. Apprendre l'historia, c'est le point central de la doctrine grégorienne de l'image religieuse » (p. 102-103).

6 Si la présence d'images à l'intérieur des églises se trouve ainsi justifiée contre les tendances iconoclastes d'un Serenus, Grégoire le Grand en limite l'usage en lui attribuant une fonction essentiellement mémorielle et didactique. Elles n'ont pas d'autre effet que des lettres au sein d'un livre d'histoire. Du même coup, la perception de l'image « est entièrement pensée sur le mode de la lecture d'un texte écrit » (p. 102). L'image ne saurait véritablement se voir. Elle doit se lire comme s'il s'agissait d'une écriture : le spectateur ne saurait s'arrêter à ce qu'il voit et à ce qu'il sent, pas plus que le lecteur ne saurait se contenter d'admirer la forme visible des lettres tracées par le 
scribe que ses yeux rencontrent sur la page. Lire implique que l'on comprenne ce que signifient ces images que sont les lettres tracées sur la page. Il en serait de même de l'image peinte. D'ailleurs, pour cela, il est généralement nécessaire de s'aider du texte qui en a permis la réalisation, en l'occurrence la Bible (ou, à défaut, du commentaire de quelqu'un qui est capable de le lire).

7 Cette "démarche réductrice » a dominé la théorie médiévale de l'image. Mais elle ne s'est pas arrêtée là. Comme le souligne J.-Cl. Schmitt, elle continue à peser «jusqu'à aujourd'hui sur notre perception des images et souvent sur les méthodes de l'histoire de l'art » (p. 102). C'est le cas, par exemple, d'Émile Mâle qui, dans L'Art religieux du XIII siècle en France, publié en 1898, comparait la cathédrale à la "Bible des pauvres ", et convoquait la "bibliothèque du Moyen Âge »-et plus particulièrement le Speculum maius de Vincent de Beauvais - pour déchiffrer les œuvres d'art dont le sens s'était obscurci et qui, au regard des spectateurs ignorant les textes de la tradition biblique, risquaient de devenir de parfaites énigmes ${ }^{1}$.

8 Pourtant, selon Grégoire le Grand, l'image ne se réduit pas à cette fonction d'illustration de l'histoire biblique ou de la théologie chrétienne à destination des laïcs. Les fidèles peuvent aussi concevoir « une ardente componction au spectacle de la scène représentée ». Le sentiment qu'ils éprouvent au moment de voir l'histoire que leur montre la peinture, par exemple la crucifixion, peut alors les inciter à vouloir adorer, non pas l'image bien sûr, mais la chose même qu'elle représente.

9 Comme le remarque J.-Cl. Schmitt, la componction que peut susciter la vision d'une image jouera un rôle de "pierre d'attente»(p.103). Son importance deviendra toujours plus grande au cours des siècles à venir, sans pour autant se séparer entièrement de la fonction principale que lui attribue Grégoire le Grand. On le constate tout d'abord par une interpolation introduite au milieu du viII ${ }^{\mathrm{e}}$ siècle dans une des deux lettres de ce pape, à laquelle J.-Cl. Schmitt accorde une valeur déterminante. L'auteur de cette interpolation commente l'envoi à son destinataire de deux panneaux représentant le Sauveur et la Vierge Marie. L'ermite désirait avoir ces peintures devant les yeux afin de pouvoir les contempler chaque jour, de sorte que son âme s'enflamme tandis que les yeux de son corps regardent l'image du Christ et de la Vierge. Le désir de l'ermite a valeur programmatique. Grâce à l'image, il est possible de démontrer les choses invisibles à l'aide des choses visibles. Cela peut évidemment s'appliquer au passé, qui est en quelque sorte devenu invisible. Mais une telle propriété ne se limite pas à l'histoire humaine, serait-elle contenue dans la Bible.

10 L'image est devenue, pour l'ermite, l'objet d'un désir intense. Ce dernier est comparé à l'amant qui souhaite ardemment voir sa bien-aimée, et la peinture de la Vierge apparaît analogue à un objet d'amour. Si son apparition est momentanée et transitoire, si elle ne donne lieu à aucune union définitive, elle n'en est pas moins une expérience réelle et la source d'une "affection» ou d'une authentique sensation de bonheur. L'émotion générée par l'image reste dépendante ici de la mémoire et de la scène dont cette dernière doit assurer l'identité et la légitimité. Mais, en permettant au spectateur de se souvenir du Fils de Dieu, l'image lui fait éprouver un sentiment qui est identifié à la chaleur de l'amour. Ainsi, tandis que la peinture, telle une écriture, fait revenir à la mémoire le Fils de Dieu, l'âme se réjouit de sa résurrection et se lamente à cause de sa passion.

11 Le rôle et le fonctionnement de l'image qui se dégage de cette interpolation se retrouvera parmi les arguments avancés par le pape Hadrien $\mathrm{I}^{\mathrm{er}}$ dans la lettre que celui- 
ci adresse en 785 à Irène et à son fils Constantin VI, empereur de Byzance, afin qu'ils renoncent à leur politique iconoclaste et rétablissent le culte des icônes au sein de l'Église grecque. Hadrien $\mathrm{I}^{\text {er }}$ commence par justifier la vénération des images en invoquant la fonction qu'elle comme mémoire de l'histoire, conformément à la position traditionnelle de Grégoire le Grand. Mais il ne s'en contente pas. Selon lui, en effet, l'image permet d'atteindre la nature invisible de la divinité. Représentant aux yeux du fidèle la forme qu'a prise le Christ lorsque celui-ci s'est incarné dans l'histoire, la peinture est une sorte d'équivalent artistique du mystère de l'incarnation; elle offre du même coup un moyen d'accéder à Dieu. Alors même qu'il a perdu avec la Chute la figure que Dieu avait imprimée en lui lorsqu'il l'a façonné - ad imaginem et similitudinem nostram, à l'image et à la semblance de Lui-même, d'après la Genèse -, l'homme pourra retrouver par l'intermédiaire de la vision d'une image sa véritable identité.

Si les commentaires médiévaux consacrés aux images ne cessent de s'appuyer sur l'autorité de Grégoire le Grand, la nouvelle conception qui se dégage de la lettre d'Hadrien $\mathrm{I}^{\text {er }}$ et qui s'affirme désormais, s'inscrit plutôt dans la tradition néoplatonicienne. Elle remonte pour l'essentiel à Plotin. C'est en effet au néoplatonisme chrétien et en particulier au pseudo-Denys l'Aréopagite (début du $\mathrm{VI}^{\mathrm{e}}$ siècle) que l'image doit la place qu'elle va progressivement prendre dans le culte divin. Dans la Hiérarchie céleste, ce dernier établit une gradation entre l'homme et Dieu qui donne aux images sensibles, portées par la lumière, un rôle intermédiaire qui permet d'objectiver le divin. Le visible est une médiation matérielle, une transition vers Dieu; il a donc une valeur théophanique. Cependant, la pensée du pseudo-Denys ne marquera véritablement la réflexion esthétique occidentale qu'à partir du XII siècle. On la rencontre en particulier chez Suger, l'abbé de Saint-Denis. Comme le souligne J.$\mathrm{Cl}$. Schmitt, ses écrits et l'église qu'il fait construire consacrent "une complète réhabilitation de l'image comme instrument de la contemplation de Dieu » (p. 100). Loin de détourner de la lecture de la Bible, comme c'est le cas chez saint Bernard (ou saint Augustin), l'attirance que suscite la beauté des décorations ornant l'église de Saint-Denis permet au contraire de se décharger des occupations terrestres et de rejoindre le monde céleste habité par Dieu. Opérant le passage d'un lieu à un autre, les ornements de l'église sont ainsi le support d'une translatio. Ils conduisent celui qui se laisse émouvoir par leur splendeur vers la source même de la lumière qui brille en eux et lui permettent de parvenir à une véritable illumination. Plutôt que de traduire le visible en identifiant la scène ou les personnages représentés, comme pourrait le faire une didascalie (ou tout autre procédé destiné à ramener l'image au texte), l'écriture fait ici référence à une expérience que la vue seule peut faire. Aucune lettre ne saurait décrire ce que voient les yeux.

L'image semble pouvoir l'emporter dorénavant sur l'écriture. Dans son Rationale divinorum officiorum, écrit à la fin du xiII ${ }^{\mathrm{e}}$ siècle, Guillaume Durand cite, à côté de la célèbre formule de Grégoire le Grand, un autre passage de ce même auteur. Celui-ci y affirme que « les représentations des choses extérieures attirent Dieu dans l'intérieur de l'âme; et, en quelque sorte, tout ce que l'on pense, en voyant de feintes images, se peint dans le cœur, s'il n'est plus vrai de dire que l'objet auquel on pense avec attention dans son cœur se peint à nos yeux d'images fictives ». Les sculptures représentées au portail des églises ouvrent ainsi un espace situé à l'intérieur du cœur même des fidèles. Par l'intermédiaire des images, c'est Dieu qui entre au plus profond de l'homme afin d'y inscrire la marque de sa présence et l'amener à se régénérer. L'œil prévaut désormais 
sur l'oreille. L'écriture (y compris l'Écriture sainte) serait une simple représentation: elle ne peut que conserver la mémoire d'un passé disparu qu'elle ne saurait autrement faire revivre. En revanche, l'image posséderait une efficacité supérieure : elle serait une authentique repraesentatio (au sens où l'entend cette fois la rhétorique à propos de l'enargeia du discours, c'est-à-dire une sorte d'évidence). C'est pourquoi elle paraît capable de toucher l'âme. Davantage que les lettres, les images parviendraient à donner accès à l'ineffable. En passant par le corps du visible, l'invisible deviendrait véritablement présent.

Les différentes images, en deux ou trois dimensions, étudiées plus précisément par J.$\mathrm{Cl}$. Schmitt, s'inscrivent toutes dans le cadre rituel et cultuel du christianisme. S'étant libérée d'une conception réductrice qui en faisait un double de l'écrit, dont pourraient se passer les clercs qui savent lire, soit une simple illustration, l'image fait pleinement partie des pratiques religieuses et politiques qui mettent en jeu le destin des individus et de la communauté à laquelle ils appartiennent, et qui impliquent aussi bien ce monde-ci que l'au-delà. J.-Cl. Schmitt rejoint ici la thèse développée par $\mathrm{H}$. Belting dans son livre paru en allemand en 1990, Image et culte. Une histoire de l'art avant l'époque de l'art. C'est grâce à l'existence d'un culte que l'image prend corps. Mais qu'en est-il en dehors de la foi chrétienne, quand ce n'est plus Dieu qui se rend présent à travers les images?

La puissance d'incarnation octroyée aux images par le christianisme médiéval est paradoxale. Pour ne pas sortir de la tradition latine afin de s'intéresser aux religions à tendance iconoclaste, il suffit de penser à ce personnage emblématique du rapport à l'image, auquel on a même attribué l'origine de la peinture: Narcisse (que mentionne rapidement J.-Cl. Schmitt à propos du rôle de l'imagination dans le discours amoureux médiéval, p. 347). Le visage qui se reflète dans le miroir de la source et dont il tombe amoureux est, comme l'écrit Ovide dans le troisième livre des Métamorphoses (v. 417-18), un espoir sans corps (spem sine corpore). Narcisse prend pour un corps (corpus putat esse) ce qui n'est en fait que de l'eau (unda) - ou qui n'est qu'une ombre (umbra, selon une variante). Il n'y a là qu'un fantôme appartenant au monde des morts, un fantasme comme ceux qui hantent le monde des rêves et qui n'a d'autre lieu que l'imagination de l'amant (celle de Narcisse, bien sûr, comme de tous les amants de la littérature médiévale dominée par Amour, dont il est la figure symbolique). "Si seulement je pouvais me séparer de notre corps!» (v. 467) s'exclame le jeune homme, qui espère ainsi donner corps - celui qu'il partage avec l'objet de son désir - à cette image inaccessible qu'il porte en lui. Il ne peut cependant que se frapper la poitrine et se laisser mourir afin de rejoindre l'ombre qui l'attend sur les rivages du Styx. Son corps est voué à disparaître. Il ne reste à sa place qu'une fleur (Nusquam corpus erat ; croceum pro corpore florem, v. 509) : une image sans corps, une métaphore.

On connaît la réponse qu'oppose Jean de Meun à l'histoire de Narcisse qui domine la première partie du Roman de la Rose, écrite par Guillaume de Lorris : c'est la réussite de Pygmalion (Métamorphose, X). Lui aussi est amoureux d'une œuvre d'art dont il est le créateur, au lieu d'une femme pourvue d'un véritable corps. À l'image peinte à la surface de l'eau succède toutefois une statue. Pygmalion n'arrive pas à croire que l'ivoire dont elle est faite ne soit un corps (v. 255) et le désir qui l'anime finira par donner vie au simulacre qui se réveille sous ses baisers. Celui-ci est devenu un corps (corpus erat, v. 283). Cette métamorphose de l'œuvre d'art, qui devient un corps vivant dont la présence est capable de répondre au désir amoureux de son créateur, peut-elle 
toutefois se réaliser autrement que par le pouvoir de la fiction, soit à l'aide d'une œuvre d'art, c'est-à-dire dans un espace fantasmatique ? À moins que Dieu n'intervienne à la place de Vénus invoquée par Pygmalion pour donner aux images qui le représentent dans l'église la vie qu'il a insufflée à Adam après l'avoir façonné dans de l'argile. Seul les miracles du christianisme semblent en effet capables de donner aux images un corps pourvu d'une véritable présence. Dès qu'on sort du cadre défini par cette religion, les images paraissent redevenir fantomatiques. Aussi lui faut-il commencer par abolir leur pouvoir maléfique.

C'est cette victoire du christianisme sur les puissances narcissiques et mortifères qui hantent les images que célèbre symboliquement le récit « Du jeune homme qui épousa l'image de pierre ", repris par Mérimée dans La Venus d'Ille et auquel J.-Cl. Schmitt consacre une dizaine de pages (p.122-131). Dans la version racontée au milieu du XIII ${ }^{\mathrm{e}}$ siècle par La Vie des Pères, l'épisode se passe à Rome au temps de Grégoire le Grand. La statue à laquelle le jeune homme passe au doigt son anneau de mariage est celle de Vénus. Elle sera finalement détruite en même temps que toutes les idoles païennes auxquelles on continuait de consacrer des cultes diaboliques. Pour que le jeune homme puisse en être libéré, il faudra l'intervention miraculeuse de la Vierge. Mais il faudra surtout réaliser à sa place une autre image : celle de la Vierge elle-même. C'est elle qui réussira à vaincre sa rivale. Grégoire le Grand apparaît ainsi, non seulement comme celui qui légitime l'existence des images religieuses alors même qu'il condamne les cultes idolâtres, mais aussi comme celui qui opère le transfert du pouvoir dont étaient pourvues les idoles antiques aux images recueillies au sein des églises. Mais tandis que les premières entrainent ceux qui se laissent séduire par leurs charmes en enfer, les secondes leur ouvrent les portes du paradis. Les unes ne sont que des ombres et n'ont de la vie que les apparences, les autres ont un corps porté par une véritable vie. Mais cela ne s'avère possible que grâce à la présence de Dieu - et du récit qui nous fait croire à ses miracles... Quel serait cependant le destin de ces images quand Dieu se retire - ou qu'on s'en passe et qu'il est réduit à n'être lui aussi que le principe d'une fiction. Gardent-elles leur corps ou deviennent-elles à leur tour des figures fantasmatiques et illusoires, à l'instar de n'importe quelle idole? Et que leur arrive-t-il quand elles ne sont plus des objets de culte au sein d'une société chrétienne, mais des "objets d'art » recueillis dans des musées comme la statue de Vénus dans le Colisée ? Le beau livre de $\mathrm{J}$.-Cl. Schmitt nous invite en tout cas à nous rappeler qu'elles ont pu avoir un corps et une véritable présence, et qu'il ne faudrait pas l'oublier quand on les étudie, ou quand on les regarde. Tel est en quelque sorte le «transfert »- à rebours - auquel il nous convie.

\section{NOTES}

1. É. Mâle, L'Art religieux du XIII e siècle en France. Étude sur l'iconographie du Moyen Âge et sur ses sources d'inspiration, Paris, 1948 [1898], cité d'après l'éd. du Livre de Poche, Paris, 1987, p. 11 et 22. 